\title{
ALLOCATION OF WORKERS OVER JOBS
}

BY

JAN TINBERGEN*

1 THE LABOUR MARKET TRANSACTIONS PROBLEM

This essay's subject matter is part of the labour market problem. As for all markets the equilibrium position is characterized by two aspects: what price will prevail and what is the volume transacted? For a complex of markets the prices and transactions of each compartment characterize equilibrium. In this essay only the transactions aspect will be discussed; it may also be called the allocation problem: which worker will be allocated which job? The number of workers will be assumed equal to the number of jobs. Here workers, as in the American and possibly other censuses, stands for all who work, independents as well as employees. Workers are characterized by personality traits, including factors such as years of schooling completed, manual dexterity, IQ, social intelligence (i.e. the ability to deal with people), etc. The number of independent personality traits is hardly known, since many concepts used may partly overlap. Jobs are characterized by a set of requirements which personality traits have to satisfy in order that the job can be done (more or less) satisfactorily. Some personality traits may be relevant to several job requirements, for instance, zeal or kindness; other personality traits may be irrelevant.

\section{THE ALLOCATION PROCESS}

The allocation of persons to jobs may take place in different ways, organized or more haphazardly. For job categories held by large numbers of persons allocation will be the subject of negotiations, conducted by organizations of employers and employees. For less frequently held jobs, personal negotiations or simply a personal choice will be decisive. In each of these processes employers or independent workers will tend to maximize profits, whereas employees or dependent workers will tend to maximize utility. Strictly speaking, profits are a proxy only for utility, but profits can be determined by calculations which facilitate the execution of negotiations. For large-scale negotiations production functions also enter the picture as helpful proxies in estimating the conse-

\footnotetext{
* Emeritus Professor of Economics, Erasmus University, Rotterdam.
} 
quences of the decisions at stake. Thus, production functions enable the economist to estimate marginal productivities of various types of workers ( $c f$. Gottschalk, 1978). It is often thought that the number of workers allocated depends on such marginal productivities and on wage rates prevailing in equilibrium. Much empirical work on production functions is available.

\section{UTILITY MAXIMIZATION}

As observed, a more precise picture of allocations should be based on utility rather than profit maximization, since the latter approach implicitly assumes that money income is the only, or main, determinant of human welfare or satisfaction, phrases we shall use as synonyms for utility. The former approach assumes that, alongside income, other aspects of a job also affect satisfaction, positively or negatively. Thus, labour disutility is an aspect considered at an early stage of the development of economic theory. Somewhat later labour satisfaction was recognized as a possibly positive determinant of welfare. Whether the two aspects result, on balance, in a positive or a negative contribution to welfare depends very much on the nature of the job discussed. It also depends on the nature of the person discussed.

As observed, the number of personality traits and job characteristics may be considerable and many of them may enter the utility or welfare function. In order to make progress in our understanding of the labour market, empirical estimation of welfare functions is important. A considerable amount of research in this area has been done by Van Praag and his school ( $c f$. Van Praag, 1973, for further references).

Another codeterminant of welfare was proposed by the present author (Tinbergen, 1956, 1959) and called 'tension.' It stands for the possible difference between the degree or level of an ability required $(h)$ and the level actually attained, $h^{\prime}$ and the assumption implies that a positive and a negative tension are equally disliked. So a proper measure may be $\left|h-h^{\prime}\right|$, or $\left(h-h^{\prime}\right)^{2}$ or even a higher power of $h-h^{\prime}$. We opt for $\left(h-h^{\prime}\right)^{2}$, where $h$ and $h^{\prime}$ vary from 1 to $H$.

Empirical data on degrees required and degrees attained are rare. Only recently Rumberger (1982) has produced a vast material on schooling required for the jobs of a large sample of the American labour force. Since actual schooling is known from the censuses, we are now able to check empirically whether allocation of workers over jobs is codetermined by schooling tension. The variables $h$ and $h^{\prime}$ will be measured in Rumberger's way, comprising six levels. Our empirical results will be discussed in section 7 , preceded by a theoretical frame (sections 4 through 6). 


\section{THE ALLOCATION MATRIX $\Phi$ AND THE ALLOCATION PROBLEM}

Simplified by the use of one aspect, schooling, only, our allocation problem may be illustrated by a matrix $\Phi$ whose elements $\varphi_{h h^{\prime}}$ represents the promilles of the labour force characterized by $h$ and $h^{\prime}$. It follows that $\Sigma_{k^{\prime}} \Sigma_{h^{\prime}} \varphi_{h^{\prime} h^{\prime}}=1000$. It is appropriate to add the borders of $\Sigma_{h} \varphi_{h h^{\prime}}=\varphi_{. h^{\prime}}$, the column totals, and $\Sigma_{h^{\prime}} \varphi_{h h^{\prime}}=$ $\varphi_{h}$, the row totals. Both borders add up to 1000 .

The matrix contains $H^{2}$ cells, which constitute a new concept needed and a smaller area than a labour market compartment. The latter will not be used in the present essay, since we are not dealing with the price aspect; but is seems useful to add a definition of compartments, in order to illustrate the difference between cells and compartments. The latter are a set of cells showing the same earnings since compartments, being the smallest units considered, are supposed to be homogeneous. A labour market with one aspect only must be represented by a one-dimensional set (a vector) of compartments, numbering $H$ in our example, and a two-dimensional set (a matrix) of cells. Already two aspects would confront us with more-than-two-dimensional sets of cells, tensors.

In addition to the definition of tension for one cell, $\left(h-h^{\prime}\right)^{2}$, we will define total tension $T=\Sigma_{h} \Sigma_{h^{\prime}} \varphi_{h h^{\prime}}\left(h-h^{\prime}\right)^{2}$.

The allocation problem consists of finding the unknowns $\varphi_{h h^{\prime}}$ from the given border vectors $\varphi_{h^{\prime}}$, the supply vector, and $\varphi_{h}$, the demand vector. As observed, we have simplified our problem by the assumption of equality of the numbers of workers $\Sigma \varphi_{. h^{\prime}}$ and of jobs $\Sigma \varphi_{h .}(=1000)$.

The number of relations that have to be satisfied equals $2 H-1$, expressing the definitions of the supply and the demand vectors, each numbering $H$, but one dependent, since $\Sigma_{h} \Sigma_{h^{\prime}}=1000$.

The number of degrees of freedom appears to be $H^{2}-2 H+1=(H-1)^{2}$.

\section{TWO PARTICULAR SOLUTIONS}

With so many degrees of freedom the number of solutions is infinitely large to the power $(H-1)^{2}$, unless more relations have to be satisfied. Among the many solutions two may be worth studying, which we will indicate by $t$ and $p$. The solution $\varphi_{h h^{\prime}}=t_{h h^{\prime}}$ stands for the so-called Northwest-corner rule, used for the solution of a transportation problem and due to Hitchcock (1941). The procedure to calculate the $t$-value may be described as follows.

Step 1. For $h=h^{\prime}=1$ (the northwest-corner of $\Phi$ ) take

$$
\varphi_{11}=\min \left(\varphi_{1}, \varphi_{.1}\right)
$$

Step 2. If $\varphi_{1}>\varphi_{.1} ; \varphi_{21}=0$ and $\varphi_{12}=\min \left(\varphi_{1 .}-\varphi_{.1}, \varphi_{.2}\right)$

$$
\text { If } \varphi_{1}<\varphi_{.1} ; \varphi_{12}=0 \text { and } \varphi_{21}=\min \left(\varphi_{.1}-\varphi_{1}, \varphi_{2}\right)
$$


Repeat the same procedure for the remaining part of $\Phi$. The solution obtained will have a minimum of nonempty cells.

In the particular case where $\varphi_{h}=\varphi_{h^{\prime}}, h=h^{\prime}$ for $1 \leqslant h \leqslant H$ (that is, the vector $\varphi_{h}=\varphi_{. h}$, or 'educational equilibrium') only the main diagonal of $\Phi$ will be nonempty; all

$$
\varphi_{h h^{\prime}}=0 \text { for } h \neq h^{\prime}
$$

By solution $p$ (symbol suggested by the word 'probability') we understand:

$$
1000 \varphi_{h h^{\prime}}=\varphi_{h \cdot} \cdot \varphi_{. h^{\prime}}
$$

The solution resembles the calculation of the probability of the combination $h$, $h^{\prime}$ if the probability of value $h$ is $\varphi_{k}$ and independent of the probability $\varphi_{h^{\prime}}$ of value $\eta^{\prime}$, both expressed in per mille.

In the particular case where all $\varphi_{h .}=\varphi_{. h^{\prime}}=1000 / H$ all $\varphi_{h h^{\prime}}$ will be equal to $1000 / H^{2}$.

The two solutions constitute opposites in the sense that $t$ concentrates all observations as closely to the main diagonal as possible whereas $p$ spreads them evenly over the square matrix $\Phi$ and so constitutes a 'minimum of concentration.'

Another case worth mentioning is the one where both vectors $\varphi_{h \text {. }}$ and $\varphi_{. h^{\prime}}$ equal the binomium $H-1$, that is, the consecutive terms of $1000(1 / 2+1 / 2)^{H-1}$, a good approximation of the Gaussian or normal distribution. Matrix $\Phi$ will then be a good approximation of a two-dimensional orthogonal normal distribution.

\section{SOLUTION $t$ IS THE MINIMUM TENSION SOLUTION}

In order to show that solution $t$ constitutes the matrix with minimum total tension $T$ as described in section 4 we introduce a small decrease $-\varepsilon$ to the frequency $\varphi_{h h^{\prime}}$ of a cell on the $t$-path $h . h^{\prime}$. In order not to violate the conditions imposed on the row and column totals we have to extend the variation to a 'variation quadruple', adding a positive increment $+\varepsilon$ somewhere into the same row and the same column of $h, h^{\prime}$, plus an additional $-\varepsilon$ to the cell $\left(h+a, h^{\prime}+a^{\prime}\right)$, if $a$ and $a^{\prime}$ are the shifts to the reference cell where the first $-\varepsilon$ is applied. The increase in total tension $T$ then may be written:

$$
\Delta T=-\varepsilon\left\{(\Delta h)^{2}+(\Delta h+\Delta a)^{2}-(\Delta h+a)^{2}-(\Delta h-a+\Delta a)^{2}\right\}
$$

where $\Delta h=h-h^{\prime}$ and $\Delta a=a-a^{\prime}$.

Execution of the calculation yields: 


$$
\Delta T=+2 \varepsilon a a^{\prime}
$$

In order to interpret this result we have to be aware of the necessity that a variation $-\varepsilon$ can only be applied to a nonempty cell, hence a $t$-cell. The two cells where $-\varepsilon$ is applied must be $t$-cells, and a third cell may, but need not, be such a cell.

With regard to the signs of $a$ and $a^{\prime}$ four combinations are possible, since both $a$ and $a^{\prime}$ can be $>0$ or $<0$. The corresponding configurations have been indicated below, where the $t$-cell started which has been italized and the variation in $\varphi_{h h^{\prime}}$ been indicated by 'pos' or 'neg' according to its sign. Below each configuration the signs of $a, a^{\prime}$ and $\Delta T$ have been added:

Configuration:

\begin{tabular}{|c|c|c|c|c|c|c|c|}
\hline \multicolumn{2}{|c|}{ I } & \multicolumn{2}{|c|}{ II } & \multicolumn{2}{|c|}{ III } & \multicolumn{2}{|c|}{ IV } \\
\hline neg & pos & pos & neg & neg & pos & pos & neg \\
\hline pos & neg & neg & pos & pos & neg & neg & pos \\
\hline$a>$ & & $\begin{array}{r}a< \\
\Delta\end{array}$ & & $\begin{array}{r}a< \\
\Delta\end{array}$ & & $a>$ & $<0$ \\
\hline
\end{tabular}

The $t$-path is characterized by movements between consecutive cells either to the right or downward (i.e., either $h$ or $h^{\prime}$ or both increase); no movements to the left or upward are permitted. Since negative changes in $\varphi_{h h^{\prime}}$ must be in $t$-cells only, configurations II and IV are impossible. That implies that variations in $\varphi_{h h^{\prime}}$ away from $t$-cells (configurations I and II) always lead to $\Delta T>0$, an increase in total tension. Solution $t$ hence constitutes the solution with minimum total tension. ${ }^{1}$

7 EMPIRICAL RELATION BETWEEN $\varphi_{h h^{\prime}}, p$ AND $t$

With the help of the empirical data on schooling required for a large sample of workers, collected and computed by Rumberger and mentioned in section 3 , we now will study the relationship between $\varphi_{h h^{\prime}}$ and $t$. The hypothesis we are going to test is that $\varphi_{h h^{\prime}}$ is a weighted average of $t$ and $p$, or

$$
\varphi_{h h^{\prime}}=\alpha p+(1-\alpha) t
$$

1 Although of no relevance to the present essay it may be added that if production per unit of frequency $\varphi_{h h^{\prime}}$ equals $\left(h+h^{\prime}\right)^{\alpha}$, or $\left(q h+q^{\prime} h^{\prime}\right)^{\alpha}$, the $t$-matrix also constitutes an extremum of production, namely a minimum if $\alpha<0$ (decreasing returns) and a maximum if $\alpha>0$ (increasing returns). 
In this relation the unknown is $\alpha$ and can be obtained from a simple correlation:

$$
\varphi_{h h^{\prime}}-t=\alpha(p-t)
$$

implying that

$$
\alpha=\Sigma(\varphi-t)(p-T) / \Sigma(p-t)^{2}
$$

A constant term needs not to be added to (7.2), since $\varphi, p$ and $t$ all represent frequencies in promilles of which the total is 1000 and hence the average $1000 / H^{2}$. We already mentioned that Rumberger used a schooling level system with $H=6$.

Rumberger uses two alternative educational groupings 1 and 2 . The results are shown in Table 1.

\begin{tabular}{|c|c|c|c|c|}
\hline Educational Groupings: & & 1 & & 2 \\
\hline Regression Equations: & $\hat{\varphi}$ & $=0.5648 p+0.4352 t$ & $\hat{\varphi}$ & $=0.6164 p+0.3836 t$ \\
\hline Standard Deviations: & & $(0.0409) \quad(0.0250)$ & & $(0.0514) \quad(0.0355)$ \\
\hline Correlation Coefficients: & $\begin{array}{l}r^{2} \\
R^{\varphi p} \\
\bar{R}^{2}{ }^{\varphi \cdot p t} \\
r_{p t}^{2} \cdot p t\end{array}$ & $\begin{array}{l}=0.8783, r_{\varphi t}^{2}=0.6914 \\
=0.9775 \\
=0.9768 \\
=0.5161\end{array}$ & $\begin{array}{l}r^{2}{ }_{p p} \\
R^{2} \\
\bar{R}^{2}{ }^{p \cdot p t} \\
r_{p t}^{2 p t}\end{array}$ & $\begin{array}{l}=0.8530, r_{\varphi t}^{2}=0.8505 \\
=0.9737 \\
=0.9730 \\
=0.6517\end{array}$ \\
\hline
\end{tabular}

TABLE 1 - REGRESSION EQUATIONS AND CORRELATION COEFFICIENTS

These results are quite positive as a test of our hypothesis. The coefficients of determination, corrected for degrees of freedom, $\bar{R}_{\varphi p t}^{2}$ are remarkably high and the significance of the values found for $\alpha$ is very high as well.

The following interpretation is offered, which contains three options.

The allocation of workers over jobs can be explained in three alternative ways. Using as explanatory variable the tension minimizing variable $t, 69$ to 85 per cent of the variance in frequency distribution $\varphi$ can be explained, as shown by the values of $r^{2} t$.

"A second interpretation is that the assumption of independence of supply and demand vectors, leading to the solution $p$, is able even to explain 85 to 88 per cent of the variance, as shown by the values of $r^{2}{ }_{\varphi p}$.

A third interpretation may be expressed by the suggestion that in the explanation of the allocation by $t$ the random disturbances characteristic for an ordinary least-squares regression practically coincide with the term $\alpha p$, since $R_{\varphi . p t}^{2}$ is very close to 1 ( 97 and 98 per cent, respectively). In fact, this is an elaboration of the first interpretation. 


\section{REFERENCES}

Gottschalk, P.T., "A Comparison of Marginal Productivity and Earnings by Occupation,' Industrial and Labor Relations Review, XXXI (1978), No. 3, pp. 368-378.

Hitchcock, F.L., 'The Distribution of a Product from Several Sources to Numerous Localities,' Journal of Math. Physics, XX (1941),pp. 224-230 (quoted in Mennes, L.B.M., et al., The Element of Space in Development Planning, Amsterdam, 1969, p. 321)

Praag, B.M.S. van, and A. Kapteyn, 'Further Evidence on the Individual Welfare Function of Income: an Empirical Investigation in the Netherlands,' European Economic Review, IV (1973), pp. 33-62 and numerous other publications to be obtained from the Economic Institute, University of Leyden, Hugo de Grootstraat 32, 2311 XK Leiden, The Netherlands.

Rumberger, R.W., material handed over to the author on November 1, 1982, University of Amsterdam.

Tinbergen, J., 'On the Theory of Income Distribution,' in: Selected Papers, Amsterdam, 1959, pp. 243-263.

Formulae (7.1) on p. 257 should read:

$$
\begin{aligned}
& \lambda_{10}=\frac{\omega_{1}}{\omega_{3}}\left(\frac{s_{1} \tau_{1}}{\sigma_{1}}-t_{1}\right) \\
& \lambda_{01}=\frac{\omega_{2}}{\omega_{3}}\left(\frac{s_{2} \tau_{2}}{\sigma_{2}}-t_{2}\right)
\end{aligned}
$$

\section{Summary}

\section{ALLOCATION OF WORKERS OVER JOBS}

Labour market supply is assumed to depend on the maximization of worker welfare. Welfare is assumed partly to depend on the 'tension' between schooling required by the job and actual schooling. Hence in the allocation of workers over jobs a tendency can be expected to minimize tension. Strict minimization produces a matrix $t$ obtained by Hitchcock's Northwest-corner rule. Recent material due to Rumberger allows an explanation of 69 to 85 per cent of frequency variance. The remaining error term can be explained by the assumption of independence of demand and supply. 